\title{
Use of Ambient lonization High-Resolution Mass Spectrometry for the Kinetic Analysis of Organic Surface Reactions
}

\author{
Rickdeb Sen, ${ }^{\dagger}$ Jorge Escorihuela, ${ }^{\dagger}$ Maarten M. J. Smulders, ${ }^{* \dagger}$ and Han Zuilhof*, ${ }^{\dagger},+$ \\ ${ }^{\dagger}$ Laboratory of Organic Chemistry, Wageningen University, Dreijenplein 8, 6703 HB Wageningen, The Netherlands \\ ${ }^{\ddagger}$ Department of Chemical and Materials Engineering, King Abdulaziz University, Jeddah, Saudi Arabia
}

\section{Supporting Information}

ABSTRACT: In contrast to homogeneous systems, studying the kinetics of organic reactions on solid surfaces remains a difficult task due to the limited availability of appropriate analysis techniques that are general, highthroughput, and capable of offering quantitative, structural surface information. Here, we demonstrate how direct analysis in real time mass spectrometry (DART-MS) complies with above considerations and can be used for determining interfacial kinetic parameters. The presented approach is based on the use of a MS tag that-in principle-allows application to other reactions. To show the potential of DART-MS, we selected the widely applied strainpromoted alkyne-azide cycloaddition (SPAAC) as a model reaction to elucidate the effects of the nanoenvironment on the interfacial reaction rate.

\section{INTRODUCTION}

Chemical reactions on solid surfaces play critical roles in many fields, such as the functionalization of devices used in catalysis, solar energy conversion, nanotechnology, and biosensing. ${ }^{1}$ The advancement of such devices is hampered by the absence of general, fast, and quantitative analytical techniques to probe the kinetics of organic reactions at the solid-liquid interface. Although interfacial reactions kinetics have been studied by different techniques in the past years, ${ }^{2-7}$ their applicability so far is limited as many of these techniques can only be used for specific functional groups, conducting surfaces, and/or require high vacuum. Accordingly, the development of rapid and easyto-use analytical techniques to measure the rates of interfacial reactions is of utmost interest.

Ambient desorption/ionization (ADI) mass spectrometry techniques offer a viable alternative to overcome the above limitations. ${ }^{8}$ Particularly for thiol-on-gold self-assembled monolayers matrix-assisted laser desorption ionization timeof-flight mass spectrometry (SAMDI) has been efficiently applied over the past decade. ${ }^{9-12}$ This technique has e.g. recently been used for high-throughput optimization of reaction conditions. ${ }^{13}$ However, this technique has its experimental limitations, such as the required use of a matrix, the concomitant restriction to fragments with $\mathrm{m} / z$ values higher than 450 . Moreover, it is limited to the cleavage of relatively weak bonds, like the $\mathrm{Au}-\mathrm{S}$ one. ${ }^{13}$ Alternatively, DART-HRMS is a promising technique ${ }^{14}$ due to its ability to detect small molecules $(m / z<400)$ without either the use of a matrix or solvents or the need for weak, noncovalent surface chemistries, unlike matrix-assisted laser desorption/ionization mass spectrometry (MALDI) and desorption ionization electrospray (DESI). ${ }^{15}$ DART-MS is an atmospheric pressure ionization
(API) technique that uses high voltage to generate hot and electronically excited $\mathrm{He}$ species $\left(2^{3} \mathrm{~S}, 19.8 \mathrm{eV}\right)$, which can simultaneously ionize and desorb surface-bound species under ambient conditions. In addition, this metastable He generates in its passage through the atmosphere also highly reactive protonated water clusters and $\mathrm{O}_{2}^{\bullet-}$ species that also react with the surface. ${ }^{16}$ As a result, DART is capable of generating ionized fragments from a very wide range of functional groups, including amides and esters. Recently, DART-MS has also been successfully applied for molecules up to $m / z 5000$, e.g., ionic liquids, ${ }^{17}$ polydimethylsiloxanes, ${ }^{18}$ and oligomers. ${ }^{19}$ The effectiveness of this method has been shown for both solution and surface substrates for routine high-throughput analysis, as analysis times vary from seconds to at most minutes. ${ }^{20}$ In recent years, this technique (Figure 1) has been successfully applied for the fast, qualitative analysis of monolayers on a wide variety of surfaces ${ }^{21-23}$ and recently for the study of gas-surface heterogeneous reactions. $^{24}$

In this work, we present for the first time the use of DARTHRMS as a general and fast analytical method to obtain quantitative kinetic insights into chemical reactions at the solid-liquid interface. As a model reaction we selected the widely used strain-promoted alkyne-azide cycloaddition (SPAAC) metal-free click reaction ${ }^{25,26}$ on a nonconducting surface. This 1,3-dipolar cycloaddition is based on the use of strained cyclooctynes ${ }^{27-32}$ and has been successfully applied for bioconjugation, ${ }^{33-37}$ in vivo imaging, ${ }^{38}$ and in the field of surface functionalization. ${ }^{39-43}$ While the kinetics of SPAAC

Received: February 3, 2016

Revised: March 17, 2016

Published: March 30, 2016 


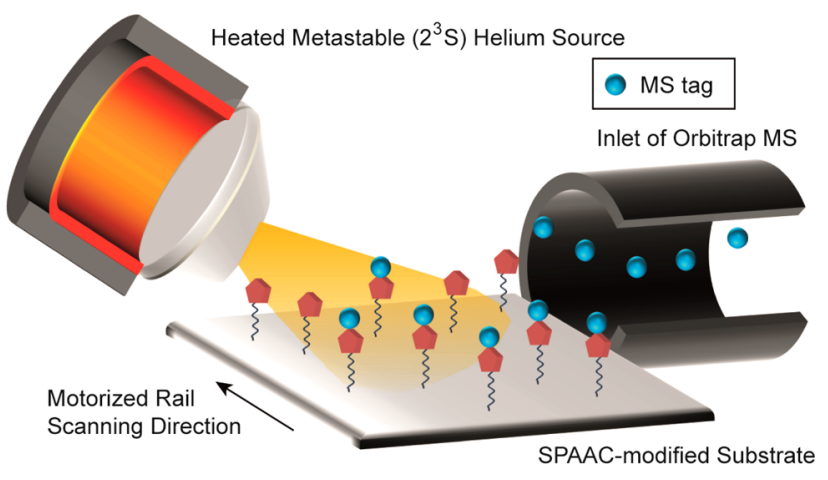

Figure 1. Schematic representation of the DART setup used for monitoring the progress of chemical reactions on surfaces.

have been well-studied in solution, ${ }^{44-46}$ no such effort has been made for the corresponding surface reaction. To this end, we investigated the reaction of a fluorine-tagged bicyclo[6.1.0]nonyne $(\mathrm{BCN})$ derivative with mixed monolayers on an aluminum oxide surface that present azide moieties in sterically different nanoenvironments in which the SPAAC reaction takes place (Scheme 1). SPAAC was particularly selected as the model reaction because it is such a well-studied reaction in solution, thereby making it an ideal model reaction to validate our new DART method for getting quantitative kinetic information of interfacial reactions.

\section{EXPERIMENTAL DETAILS}

Materials. Unless otherwise specified, all chemicals were used as received without further purification. Octylphosphonic acid, hexadecylphosphonic acid, hydrochloric acid, methanol, hexane, acetone, dichloromethane, and 2-propanol were purchased from Sigma-Aldrich. 12-Azidododecylphosphonic acid and dodecylphosphonic acid were purchased from SiKEMIA. Aluminum pieces (99.5\% purity, mirror polished, Staalmarkt Beuningen BV) were cut using a mechanical cutter into pieces of $2 \times 1 \mathrm{~cm}$. For surface modification reactions, the samples were loaded onto a specially constructed PTFE wafer holder able to hold up to 16 samples at a time thus ensuring rigorous reproducibility between samples (Figure S13).

General Procedure for the Preparation of Phosphonic Acid Monolayers. The $2 \times 1 \mathrm{~cm} \mathrm{Al} \mathrm{slides} \mathrm{were} \mathrm{sonicated} \mathrm{in} \mathrm{hexane} \mathrm{for} 15$ min followed by wiping with lint-free cotton swabs (Texwipe, Kernersville, NC) to remove polymer protection layer on top and remove any residual glue. The surfaces were chemically activated by immersing in $1: 1(\mathrm{v} / \mathrm{v}) 37 \% \mathrm{HCl}-\mathrm{MeOH}$ mixture for $5 \mathrm{~min}$, followed by washing with water and 2-propanol. The activated surfaces were then immersed into $\mathrm{N}_{2}$-filled vials containing a $5 \mathrm{mM}$ solution in 2propanol of the appropriate alkylphosphonic acid (with $\mathrm{C}_{8}, \mathrm{C}_{12}$, or $\mathrm{C}_{16}$ alkyl chain) and 12-azidododecylphosphonic acid, in the required ratio, which was varied from 0:1 up to 9:1. The solution was heated to $50{ }^{\circ} \mathrm{C}$ for $5 \mathrm{~min}$ and then left undisturbed for $5 \mathrm{~h}$ at room temperature to obtain self-assembled mixed monolayers. Then surfaces were taken out and sonicated successively for $5 \mathrm{~min}$ with 2-propanol, acetone, and $\mathrm{CH}_{2} \mathrm{Cl}_{2}$. The surfaces were then cleaned with $\mathrm{CH}_{2} \mathrm{Cl}_{2}$, air-dried, and stored under $\mathrm{N}_{2}$ atmosphere.

General Procedure for Interfacial SPAAC Reaction. Azidefunctionalized slides were immersed in a $3 \mathrm{mM}$ solution of 1 in dichloroethane under a $\mathrm{N}_{2}$ atmosphere and allowed to react. After completion of reaction for the desired time, the $\mathrm{Al}$ pieces were taken out, sonicated, and washed with acetone to remove physisorbed moieties, dried under a $\mathrm{N}_{2}$ stream, and immediately used for XPS and DART analysis.

Determination of Activation Parameters. The activation parameters (enthalpy of activation $\left(\Delta H^{\ddagger}\right)$ and entropy of activation $\left.\left(\Delta S^{\ddagger}\right)\right)$ were obtained using the Eyring equation by a least-squares fit of plots of $\ln (k / T)$ versus $1 / T$. Values for $\Delta H^{\ddagger}$ and $\Delta S^{\ddagger}$ were calculated from the slope and intercept of the aforementioned plot (eq $1)$.

$$
\ln (k / T)=\ln \left(K k_{\mathrm{B}} / h\right)-\Delta H^{\ddagger} / R T+\Delta S^{\ddagger} / R
$$

where $k$ is the observed rate constant (in $\mathrm{s}^{-1}$ ), $T$ is the absolute temperature (in $\mathrm{K}$ ), $K$ is the transmission coefficient (set to be 1 ), $k_{\mathrm{B}}$ is Boltzmann's constant, $h$ is Planck's constant, $R$ is the gas constant, $\Delta S^{\ddagger}$ is the entropy of activation (in $\left.\mathrm{J}(\mathrm{K} \mathrm{mol})^{-1}\right)$, and $\Delta H^{\ddagger}$ is the enthalpy of activation (in $\mathrm{J} \mathrm{mol}^{-1}$ ).

Analytical Instruments. Static Water Contact Angle (SCA) Measurements. The wettability of the modified surfaces was determined by automated static water contact angle measurements with a Krüss DSA 100 goniometer (volume of the drop of deionized water was $3.0 \mu \mathrm{L}$ ). The reported values are the average of at least five droplets, and the relative error is less than $\pm 1^{\circ}$.

Infrared Reflection-Absorption Spectroscopy (IRRAS). IRRAS spectra were obtained with a Bruker Tensor 27 FT-IR spectrometer equipped with a liquid nitrogen cooled MCT-detector, using a commercial variable-angle reflection unit (Auto Seagull, Harrick Scientific). A Harrick grid polarizer was installed in front of the

Scheme 1. SPAAC Reaction of BCN Derivative 1 with Immobilized Azides Present in Three Different Nanoenvironments: Freely Accessible $(n=0, \mathrm{C} 8)$, Partially Accessible $(n=4, \mathrm{C12})$, and Buried $(n=8, \mathrm{C16})^{a}$

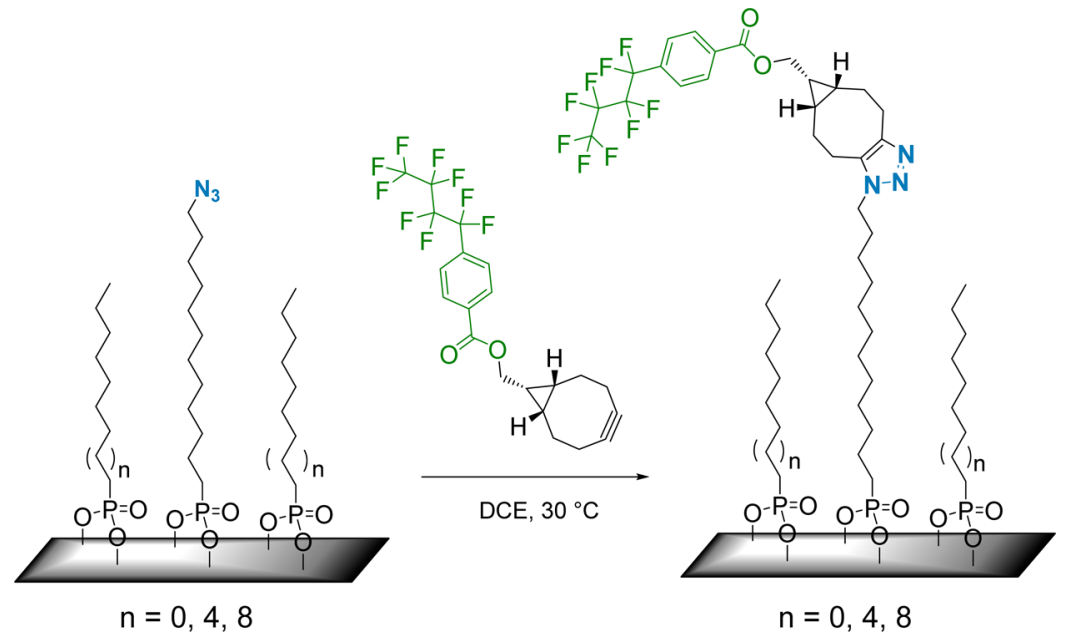

${ }^{a}$ The MS tag $\left(\left[\mathrm{C}_{11} \mathrm{H}_{4} \mathrm{O}_{2} \mathrm{~F}_{9}\right]^{-}\right)$is highlighted in green. 
detector and was used for measuring spectra with p-polarized radiation with respect to the plane of incidence at the sample surface. A Ge ATR crystal was used to obtain the spectra. Single channel spectra were collected at $35^{\circ}$ using 256 scans in each measurement. The raw data were divided by the spectrum recorded on a freshly etched reference $\mathrm{Al}$ oxide surface, after which a baseline correction was applied to give the reported spectra.

X-ray Photoelectron Spectroscopy (XPS) Measurements. The XPS analysis of surfaces was performed using a JPS-9200 photoelectron spectrometer (JEOL, Japan). Survey and high-resolution spectra were obtained under UHV conditions using monochromatic $\mathrm{Al} \mathrm{K} \alpha \mathrm{X}$-ray radiation at $12 \mathrm{kV}$ and $20 \mathrm{~mA}$ and an analyzer pass energy of $50 \mathrm{eV}$ for wide scans and $10 \mathrm{eV}$ for narrow scans. The emitted electrons were collected at $10^{\circ}$ from the surface normal with charge compensation set at $2.8 \mathrm{eV}$ and a filament current of $4.8 \mathrm{~A}$ because of the electrostatic charging in the positive direction on the surface. All XPS spectra were evaluated by using CasaXPS software (version 2.3.15). Survey spectra were corrected with linear background before fitting, whereas highresolution spectra were corrected with linear background. Atomic area ratios were determined after a baseline correction and normalizing the peak area ratios by the corresponding atomic sensitivity factors (1.00 for $\mathrm{C} 1 \mathrm{~s}, 1.80$ for $\mathrm{N} 1 \mathrm{~s}, 2.93$ for O 1s, 4.43 for F 1s, 1.18 for P 2s, and 0.54 for Al 2p).

DART-HRMS Measurements. Analysis of the SPAAC reacted Al surfaces were performed using a DART-SVP ion source (Ion-Sense, Saugus, MA) coupled to an Exactive orbitrap high-resolution mass spectrometer (Thermo Fisher Scientific, San Jose, CA), mounted on a motorized rail traveling at $0.2 \mathrm{~mm} / \mathrm{s}$. Thermo Scientific Xcalibur software (V2.1.0.1139) was used for data acquisition and processing. The measurements were performed in negative mode at $450{ }^{\circ} \mathrm{C}$ using a scan range of $\mathrm{m} / z$ 330.0-350.0 and a mass resolution of 100000 (fwhm) at a scan rate of $1 \mathrm{~Hz}$. The ion trap was tuned with a $0.1 \mathrm{mg} /$ $\mathrm{mL}$ methanolic solution of quinine $(\mathrm{m} / z 323.41$ in negative mode) to optimize capillary temperature and voltage (Figure S16). The DART source was positioned $6.1 \mathrm{~cm}$ on the horizontal scale, $7 \mathrm{~cm}$ on the vertical scale with an angle of $45^{\circ}$, such that it is around $1 \mathrm{~mm}$ above the surface (Figures S13-S16). The distance from the surface to the ceramic tube is minimized by placing them at the edge of the moving rail so that maximum of the $p-\mathrm{C}_{4} \mathrm{~F}_{9}$ benzoate ions would enter the MS.

\section{RESULTS AND DISCUSSION}

Monolayer Formation and Characterization. The proposed strategy to control the steric nanoenvironment around the azide moieties on the monolayers relies on the introduction of nonreactive alkyl chains of variable length around the reactive azide, which was linked to the surface using a $\mathrm{C}_{12}$ linker, as schematically depicted in Figure 2. In this regard, with a $\mathrm{C}_{8}$ alkyl chain (C8), the azide is freely accessible

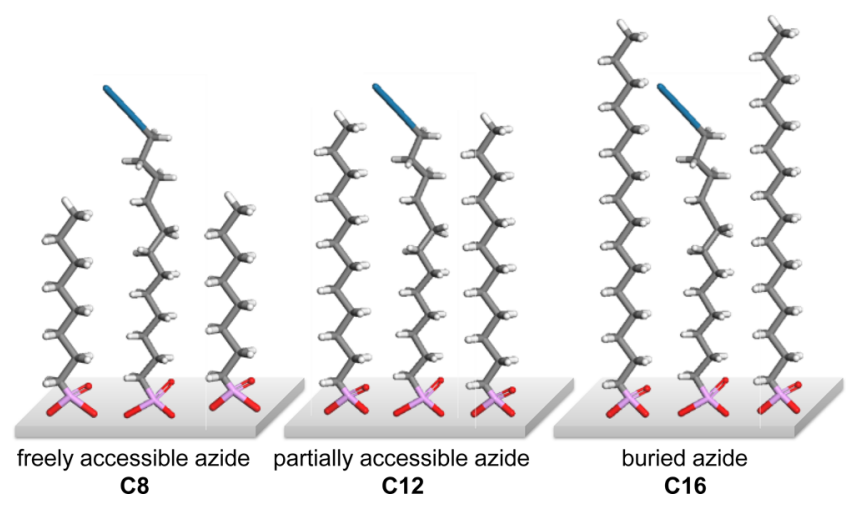

Figure 2. Schematic model of the mixed monolayer used for the SPAAC with immobilized azide groups present in the three different nanoenvironments: freely accessible (C8), partially accessible (C12), and buried (C16). layer, whereas with a longer alkyl chain, the reactive group is only partially accessible (C12) or is buried within the monolayer (C16).

For surface modification, a well-established, robust attachment chemistry relying on phosphonic acids (PAs) was used to covalently anchor the monolayer onto hydroxylated aluminum oxide surfaces. ${ }^{4-49}$ Freshly etched aluminum substrates were immersed for $5 \mathrm{~h}$ in 2-propanol solutions of 12-azidododecyl PA and the appropriate alkyl-terminated PA (in different ratios), yielding monolayers with $28-30 \% \mathrm{C}$ content as determined by XPS (Figure S1). From static water contact angle (SCA) measurements, it was found that the reaction was complete after $5 \mathrm{~h}$ (Figure S4). Remarkably, substantially longer reaction times $(16 \mathrm{~h})$ contributed to the formation of undesirable multilayers $(42-44 \% \mathrm{C})$. Contrary to previous reports, ${ }^{50-52}$ the azide content in a mixed monolayer was found to be identical to the content in the solution (within experimental error) as confirmed by XPS (Figure S2). After reaction with the $\mathrm{PA}$ mixture, the static water contact angle (SCA) increased from $35^{\circ}$ (-OH terminated aluminum oxide) to $106^{\circ}$ due to the hydrophobic nature of the alkyl chain (Table S1 and Figure S3), in accordance with similar PA monolayers on aluminum oxide surfaces. ${ }^{53}$ The XPS $\mathrm{N}$ 1s narrow scan showed two bands with a ratio $2: 1$ (399.6 and $403.4 \mathrm{eV}$, respectively), in accordance with the presence of an $\mathrm{N}_{3}$ group (Figure 3a), ${ }^{54}$ and IRRAS confirmed the presence of the $\mathrm{N}_{3}$ group (Figure $3 \mathrm{c}$ ), showing the characteristic azide peak at $2102 \mathrm{~cm}^{-1}$. 55 The XPS C 1s narrow scan (Figure $3 \mathrm{~b}$ ) shows the peak deconvolution into different components corresponding to the carbon atoms having different environments in the mixed 3:1 C8 monolayer.

IRRAS gives evidence for the presence of different functional groups in the monolayer and also provides information about the mode of attachment of the monolayer onto the surface. The positions of the antisymmetric and symmetric methylene stretching ( $\nu_{\mathrm{a}}$ and $\nu_{\mathrm{s}} \mathrm{CH}_{2}$, respectively) can be used to distinguish between monolayers with a short-range order $\left(2918 / 2850 \mathrm{~cm}^{-1}\right)$ or disorder $\left(2928 / 2854 \mathrm{~cm}^{-1}\right)$. The presence of sharp signals for the antisymmetric $\mathrm{C}-\mathrm{H}$ stretch vibration (Figure 3c) at $2925 \mathrm{~cm}^{-1}$ (C8), $2922 \mathrm{~cm}^{-1}$ (C12), and $2924 \mathrm{~cm}^{-1}$ (C16) and for the symmetric $\mathrm{C}-\mathrm{H}$ stretch vibration at $2854 \mathrm{~cm}^{-1}$ (C8), $2852 \mathrm{~cm}^{-1}(\mathbf{C 1 2})$, and $2953 \mathrm{~cm}^{-1}$ (C16), in combination with the XPS and contact angle data above, indicates the formation of densely packed monolayers without a high degree of short-range order. ${ }^{56}$

SPAAC Reaction Development. For the study of the SPAAC reaction, the endo-BCN diastereomer (1, Scheme 1) was selected. The design of the $\mathrm{BCN}$ derivative was guided by two criteria: first (part of) the molecule should be detectable by DART, and second the molecule should also be observable by XPS (for independent validation of the DART results). Previous research in our group showed DART to be effective in cleaving ester bonds both in solution and on surfaces to release a suitable MS tag that can be detected in negative ion mode (Figures S7 and S8). ${ }^{23}$ Thus, the ester bond was selected as a labile linker that couples the reactive $\mathrm{BCN}$ group with a MS tag, which was chosen to be a para-substituted benzoic acid derivative with a fluorinated butyl chain (highlighted in green in Scheme 1). Such a labeling approach has been widely studied; ${ }^{57,58}$ however, to the best of our knowledge, this is the first time an ionizable MS tag is used for interfacial kinetics elucidation. This design has three additional advantages: (1) the fluorinated alkyl chain increases the volatility of the benzoic 
(a)

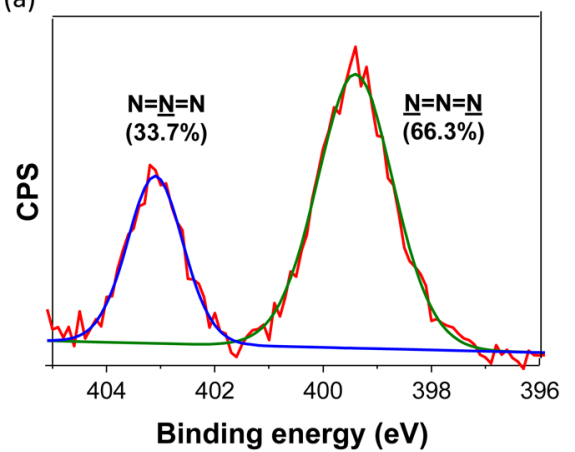

(b)

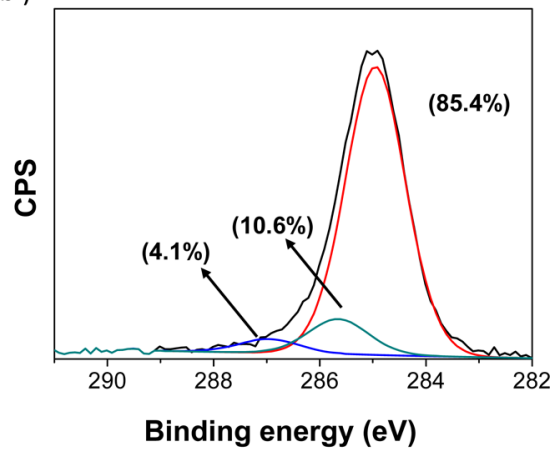

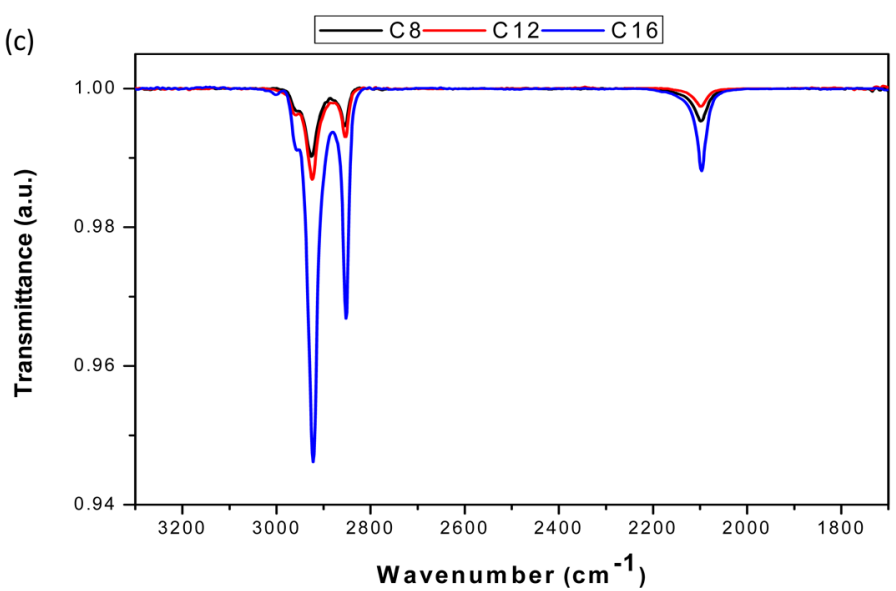

Figure 3. High-resolution XPS N 1s (a) and C 1s (b) spectra of a 3:1 mixture of octylphosphonic and 12-azidododecylphosphonic acid monolayer (C8) onto oxidized aluminum. (c) IRRAS spectra of 3:1 mixed monolayers of C8, C12, and C16 onto oxidized aluminum. The antisymmetric and symmetric $\mathrm{CH}_{2}$ stretching vibrations are visible between $2925-2922 \mathrm{~cm}^{-1}$ and $2854-2852 \mathrm{~cm}^{-1}$, respectively, while the characteristic azide peak appears at $2102 \mathrm{~cm}^{-1}$.
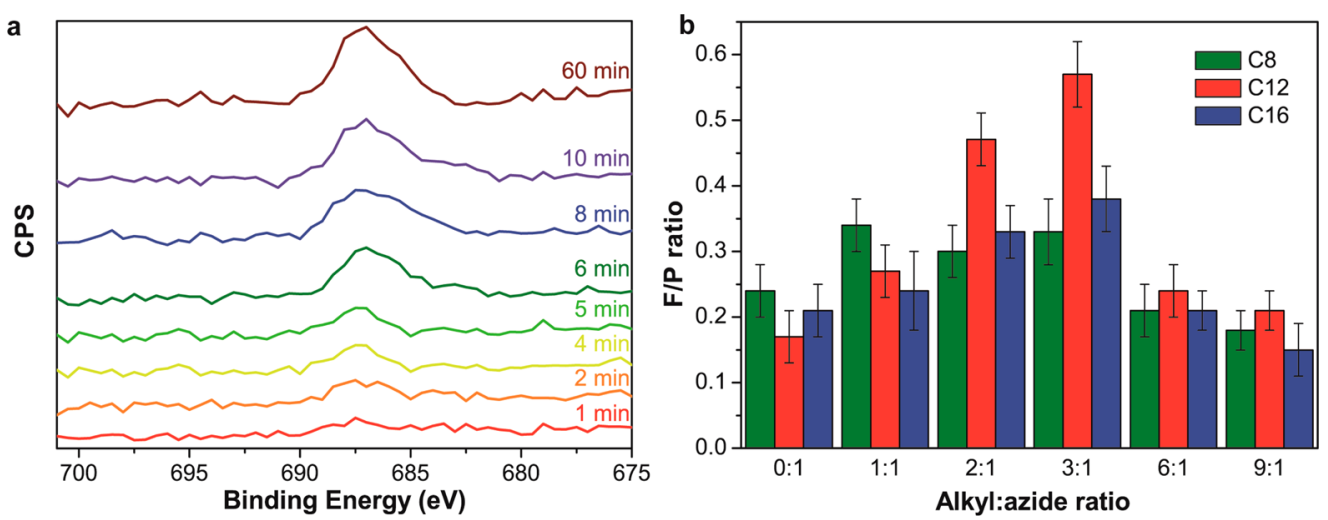

Figure 4. (a) XPS F 1s spectra after SPAAC at different reaction times. (b) F/P ratio for the three different mixed monolayers with varying C-alkyl environment after SPAAC reaction (60 min; as in rest of the paper: all results from 6-fold repetition).

acid group, allowing a rapid surface desorption; (2) the electron-withdrawing perfluorobutyl group weakens the ester bond and stabilizes the carboxylate anion; (3) F signals are easily detected by XPS, thus providing a label for an independent quantitative analysis.

The SPAAC reaction was performed using a $3 \mathrm{mM}$ solution of $\mathrm{BCN}$ derivative $(\mathbf{1})$ on the azide-terminated monolayer in dichloroethane (DCE) at $30{ }^{\circ} \mathrm{C}$. Under these conditions, the $\mathrm{BCN}$ derivative is present in large excess over the immobilized azides; therefore, pseudo-first-order kinetics can be assumed. After reaction with 1, XPS measurements showed a significant increase in $\mathrm{F}$ content on the surface (Figure 4a). As control experiment, treating $100 \%$ alkyl-terminated surfaces with a 3 $\mathrm{mM}$ solution of $\mathbf{1}$ for $24 \mathrm{~h}$ yielded no incorporation of fluorine, as determined by XPS. It is worth mentioning that no F signal was detected in a typical F 1s narrow scan, and XPS wide scans were carried out under more sensitive conditions to obtain an acceptable signal-to-noise $(\mathrm{S} / \mathrm{N})$ ratio for the $\mathrm{F}$ 1s region (Figure 4a).

In order to study the optimal nanoenvironment for the SPAAC reaction, different monolayers with varying alkyl:azide ratios (from 0:1 to 9:1) were prepared and reacted with the $\mathrm{BCN}$ derivative for $60 \mathrm{~min}$. Results clearly showed that increasing the spacing between the azide groups gave a 
substantial improvement in $\mathrm{BCN}$ incorporation: despite the reduction of $50 \%$ of azides on the surface, the $\mathrm{F} / \mathrm{P}$ ratio clearly increased when going from $0: 1$ to $1: 1$ (Figure $4 b$ ). Further variation afforded a maximum surface coverage after $60 \mathrm{~min}$ reaction time for the 3:1 monolayer (for the more diluted surfaces it was possible to reach higher conversion only at longer reaction times). Interestingly, the 3:1 ratio showed that the incorporation of the $\mathrm{BCN}$ derivative was greatest for the slightly accessible C12 monolayer, followed by the buried C16 and the accessible C8. In view of these results, the 3:1 mixed monolayer was selected for all further kinetics studies.

In the XPS N 1s spectrum of the 3:1 mixed monolayers, signals corresponding to both the azide and the triazole moiety were observed, showing the presence of the unreacted azides even after long reaction times, as also confirmed by IRRAS (Figure S9). In particular, the maximum $\mathrm{F} / \mathrm{N}$ ratio was around 1 , showing that only one-third of the azide groups had been converted. This finding was corroborated for all three monolayers by separate experiments in which the reaction was continued for 5 days and is consistent with other incomplete conversions reported in the literature. ${ }^{59}$ We may attribute this incomplete conversion primarily to steric hindrance provided by the $\mathrm{BCN}$ moiety and the attached fluorinated tail.

DART-Based Reaction Rate and Activation Parameters Determination. Despite its applicability for routine surface analysis, XPS is not a very suitable technique to determine reaction rates as a consequence of the timeconsuming data acquisition, as a typical narrow scan requires several hours (total measurement time for hexaplet data point was typically $16 \mathrm{~h}$ ). Additionally, the comparatively low sensitivity hampers study at small conversions, requiring in our case at least 6-fold repetitions to obtain usefully small experimental errors (Figure 4b). Alternatively, DART-HRMS is an atmospheric pressure ionization technique with high sensitivity and intrinsically a much higher $\mathrm{S} / \mathrm{N}$ ratio than XPS, which allows fast measurements, in particular compared to $\mathrm{XPS}$, as analysis times vary from seconds to minutes (typical total measurement time for a hexaplet data point was $18 \mathrm{~min}$ ). Under the studied conditions, the use of high resolution in the MS analysis was found to be necessary because of the presence of unassignable contaminating species (e.g., from the ambient atmosphere) with near-identical masses (Figure S17). A closer inspection of the mass spectra obtained during our measurements revealed unassigned signals for a minority of samples, close to the ion of interest (within $30 \mathrm{mu}$ ). Therefore, to rule out any contributions of these contaminants and ensure to be analyzing only the correct ion of interest, HR-MS was needed and used throughout (10 mu mass window). To appreciate the strength of DART-HRMS, it is instructive to consider the minute amount of tag that is reacted on the surface and is desorbed from it: surface concentrations as low as $\sim 1 \mathrm{pmol}$ $\mathrm{cm}^{-2}$ can be measured without the requirement of sample pretreatment. $^{24}$

Figure 5a shows a typical time-dependent mass spectrometric signal recorded for a SPAAC-modified Al slide, where the extracted ion chromatogram (EIC) of the labile group is shown in the red trace and was quantified by integrating the peak area over a fixed time interval (in gray). The intensity of MS tag $\left(\left[\mathrm{C}_{11} \mathrm{H}_{4} \mathrm{O}_{2} \mathrm{~F}_{9}\right]^{-}\right)$monitored by DART over time is assumed to be directly proportional to the extent of reaction, as this tag can only be on the surface as the result of the reaction between surface-bound azide and solution-based BCN 1. Control
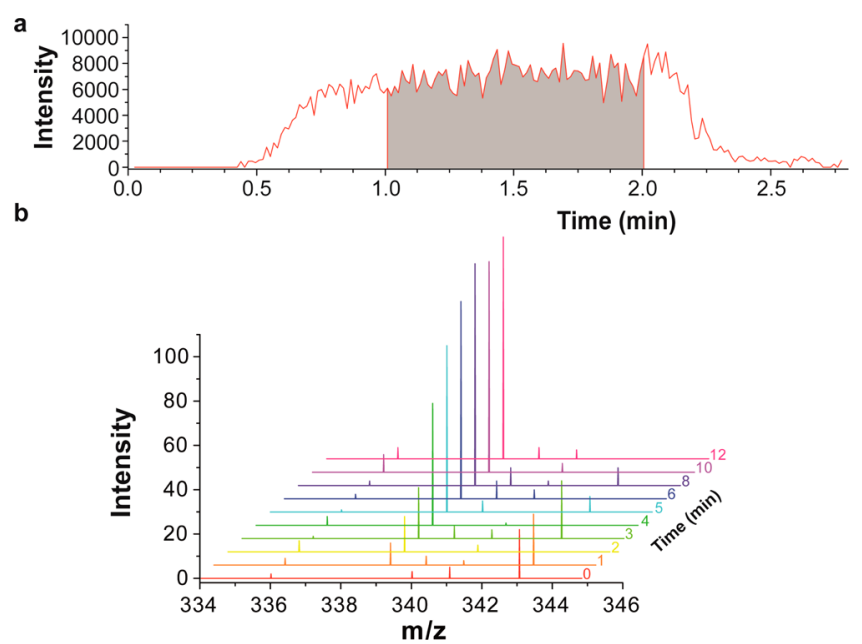

Figure 5. (a) Extracted ion chromatogram $(\mathrm{m} / z$ 339.0052-339.0072) from the labile group $\left(\left[\mathrm{C}_{11} \mathrm{H}_{4} \mathrm{O}_{2} \mathrm{~F}_{9}\right]^{-}\right)$and integrated peak area (in gray). (b) Mass spectra for the incorporation of $\mathrm{BCN}$ compound 1 to the azide-functionalized surface $\mathbf{C 1 2}$ at different times (from 0 to 12 $\min )$.

measurements without azide moieties showed no physisorption of unreacted BCN molecules onto the surface. A great advantage of DART-HRMS is the much higher sensitivity compared to e.g. XPS: while with XPS it proved challenging to obtain discernible $F$ signals for lower reaction times, with DART-HRMS intense signals with excellent $\mathrm{S} / \mathrm{N}$ ratios could be obtained routinely for reaction times as short as $15 \mathrm{~s}(\sim 3 \%$ conversion, Figure $5 b$ ).

A kinetic study was performed for each of the three mixed monolayers (C8, C12, and C16) using the intensity of the MS tag obtained by DART analysis as a measure for the conversion. In accordance with a first-order process, the rate of $\mathrm{BCN}$ incorporation (due to SPAAC) became progressively slower with increasing time, converging to a limiting value, which was different for each monolayer (Figure 6a). Particularly, the extent of the reaction was highest for the $\mathbf{C 1 2}$ monolayer, in line with the results of the XPS measurements previously discussed (Figure $6 \mathrm{~b}$ ). The lower extent of $\mathrm{BCN}$ incorporation in the case of the $\mathbf{C 8}$ monolayer can be rationalized by considering that for this system the $\mathrm{BCN}$-containing alkyl chain is free to move above the nanoenvironment created by the octyl chains, meaning that this bulky group can fold back on the monolayer, thus blocking access to unreacted azide groups in its vicinity, which can thus account for the lowest incorporation for the C8 monolayer. This freedom is reduced in the case of the C12 monolayer, where the $\mathrm{BCN}$ is forced to stand more upright on the surface. Similarly, the lower extent of BCN incorporation found for the C16 nanoenvironment can be explained by the fact that the SPAAC reaction needs to occur inside the nanoenvironment of the long hexadecyl chains, which means that as the reaction progresses the monolayer density will increase as a result of incorporation of the bulky $\mathrm{BCN}$ group within the monolayer. This can make it impossible for incoming $\mathrm{BCN}$ molecules to react with remaining azide groups that are buried within the $\mathbf{C} 16$ monolayer.

The first-order rate constants $\left(k^{\prime}\right)$ were obtained from the slope of plotting $\ln \left[\left(I_{\text {inf }}-I_{0}\right) /\left(I_{\text {inf }}-I_{t}\right)\right]$ as a function of time (Figure $6 \mathrm{~b}$ and Table 1 ). In the case under study, $I_{\text {inf }}$ is the asymptotic (integrated) EIC intensity that is reached as the reaction progress stops, and this was determined by curve 

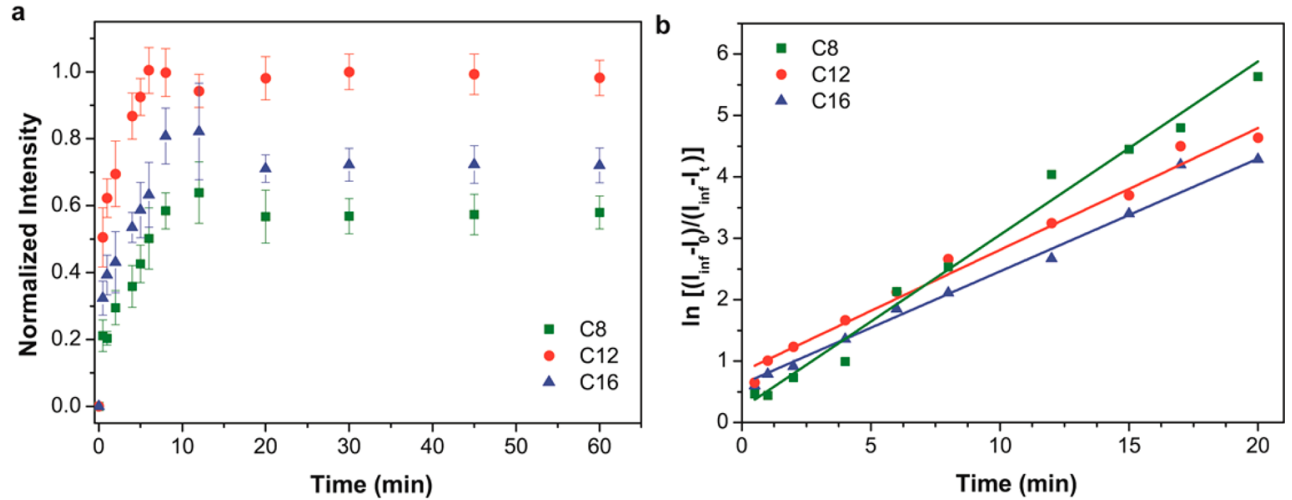

Figure 6. (a) Normalized intensity (with respect to C12) for the SPAAC reaction of BCN derivative 1 with immobilized azides in the three nanoenvironments at $30^{\circ} \mathrm{C}$ (each data point from 6-fold repetition). (b) Plot showing $\ln \left[\left(I_{\text {inf }}-I_{0}\right) /\left(I_{\text {inf }}-I_{t}\right)\right]$ vs time, used to obtain the first-order rate constants.

Table 1. First-Order Rate Constants and Activation Parameters (Determined by DART-HRMS) for the Interfacial SPAAC Reaction of BCN and Azido Moieties on the Three Nanoenvironments (Results from Sixfold Repetitions)

\begin{tabular}{ccccc} 
Monolayer & $\begin{array}{c}k_{\text {DART }}^{\prime} \\
\left(\times 10^{-3} \mathrm{~s}^{-1}\right)\end{array}$ & $\begin{array}{c}k_{\mathrm{XPS}}^{\prime} \\
\left(\times 10^{-3} \mathrm{~s}^{-1}\right)\end{array}$ & $\begin{array}{c}\Delta H^{\ddagger} \\
\left(\mathrm{kJ} \mathrm{mol}^{-1}\right)\end{array}$ & $\begin{array}{c}\Delta S^{\ddagger}\left(\times 10^{2} \mathrm{~J}\right. \\
\left.\mathrm{K}^{-1} \mathrm{~mol}^{-1}\right)\end{array}$ \\
C8 & $5.7 \pm 0.4$ & $5.3 \pm 0.3$ & $12 \pm 1$ & $-2.5 \pm 0.3$ \\
C12 & $3.7 \pm 0.3$ & $3.3 \pm 0.4$ & $24 \pm 2$ & $-2.1 \pm 1.0$ \\
C16 & $3.1 \pm 0.3$ & $2.8 \pm 0.5$ & $21 \pm 3$ & $-2.2 \pm 1.0$ \\
\hline
\end{tabular}

fitting of the data to an exponential (decay) function. For monolayers presenting freely accessible azide groups (C8), the rate constant at $30{ }^{\circ} \mathrm{C}$ was $0.0057 \mathrm{~s}^{-1}$. However, for the mixed monolayers presenting partially accessible (C12), or buried (C16) azide groups, the rate constants were 0.0037 and 0.0031 $\mathrm{s}^{-1}$, respectively, ca. 2-3-fold lower than the monolayer presenting the azide in a more accessible environment. From this, it can be concluded that as the azide becomes sterically less accessible, the reaction rate decreases, which can understood by considering that the large $\mathrm{BCN}$ derivative has to diffuse toward its azide counterpart in order for the reaction to occur. It is thus worth noting that the effect of nanoenvironment on the SPAAC reaction as could be elucidated by DART-HRMS is manifested in different ways for the rate of the reaction and for the extent of the reaction. Whereas the rate simply decreases upon increasing the steric bulk around the azide group (see Figure 7 for a schematic representation), for the extent of the reaction an optimum was found for the intermediate $\mathbf{C 1 2}$ monolayer.

It is worth mentioning that we observed relatively small differences between rate constants for the different nano- environments. While this observation is by itself already interesting from a mechanistic point of view, it-importantly-also clearly illustrates the potential of our DART measurements: we can accurately and reliably observe such small differences that are with conventional approaches, like XPS, well beyond the limits of observation. The validity of the DART method was confirmed independently by XPS (Figures S10 and S11), which yielded similar rate constants for the three monolayers. This shows the potential use of DART-HRMS as a rapid and easy-to-use tool for the accurate measurement of the rates of surface-bound reactions.

To further illustrate the convenience of DART-HRMS, the effect of temperature on the reaction rates was studied at four different temperatures in the range of $20-50{ }^{\circ} \mathrm{C}$, allowing us to extract the activation parameters by means of an Eyring analysis (Table S2). Whereas the entropy of activation is basically the same for all three cases within experimental error, the enthalpy of activation varies significantly in dependence of the nanoenvironment. In line with previously reported results, ${ }^{50}$ we observed that for the unhindered C8 monolayer the lowest enthalpy of activation $\left(12 \pm 1 \mathrm{~kJ} \mathrm{~mol}^{-1}\right)$ was found, which can be understood by an optimal overlap between the two $\pi$ systems involved in the $[3+2]$ cycloaddition during the transition state for the $\mathbf{C 8}$ system. For both the $\mathbf{C 1 2}$ and the C16 monolayers significantly higher enthalpies of activation were determined $\left(24 \pm 2\right.$ and $21 \pm 3 \mathrm{~kJ} \mathrm{~mol}^{-1}$, respectively). Apparently, the increased sterics of the reaction with higher surrounding alkyl chains are quite significant in this reaction. Two factors likely contribute to this, namely, the reduced flexibility in the orientation of the azide moieties and the increased bulk close to the monolayer surface due to the $\mathrm{BCN}$ ring and its freely rotating fluoroalkyl tail (Figure 7). These

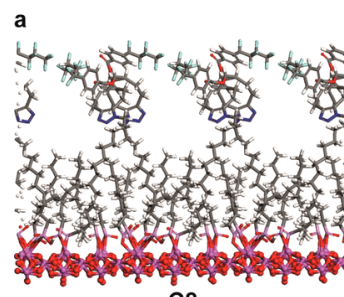

C8 b c

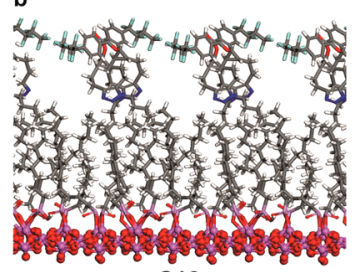

C12



C16

Figure 7. General schematic representation of the three monolayers after the SPAAC reaction: (a) freely accessible C8, (b) partially accessible C12, and (c) buried C16. 
values are all slightly smaller than the theoretically determined activation energies of $27-31 \mathrm{~kJ} \mathrm{~mol}^{-1}$. 60

While the mechanistic insights into the SPAAC reaction are relevant by themselves, it should be emphasized that these insights are the direct consequence of the availability of DARTHRMS as a fast, quantitative surface analysis technique. Further studies on the application of DART-HRMS for determining the interfacial kinetic parameters of other click reactions are ongoing in our laboratory.

\section{CONCLUSIONS}

The successful unravelling of the effects of the nanoenvironment on the rate of the commonly employed SPAAC highlights the power of DART-HRMS as a general and fast technique to study interfacial reaction kinetics in great detail. In addition, it has been shown that tailor-made control over the nanoenvironment of the reactive moieties via tuning of the accessibility of the immobilized azide within the monolayer can steer both the reaction rates and the extent of the surface-bound SPAAC reaction. We anticipate DART-HRMS provides a powerful analytical platform for the kinetic study of many other reactions on surfaces.

\section{ASSOCIATED CONTENT}

\section{S Supporting Information}

The Supporting Information is available free of charge on the ACS Publications website at DOI: 10.1021/acs.langmuir.6b00427.

Detailed experimental procedures and additional XPS, IRRAS, NMR, and contact angle data; Figures S1-S17 and Tables S1, S2 (PDF)

\section{AUTHOR INFORMATION}

\section{Corresponding Authors}

*E-mail maarten.smulders@wur.nl (M.M.J.S.).

*E-mail han.zuilhof@wur.nl (H.Z.).

\section{Author Contributions}

R.S. and J.E. contributed equally.

Notes

The authors declare no competing financial interest.

\section{ACKNOWLEDGMENTS}

The authors thank The Netherlands Organization for Scientific Research (NWO) for funding via ECHO project number 712.012.006 and VENI grant 722.012.005 and Judith Firet, Ton Marcelis, Floris van Delft, and Michel Nielen for practical and conceptual assistance.

\section{REFERENCES}

(1) Yates, J. T., Jr.; Campbell, C. T. Proc. Surface Chemistry: Key to Control and Advance Myriad Technologies. Proc. Natl. Acad. Sci. U. S. A. 2011, 108, 911-916.

(2) Gawalt, E. S.; Mrksich, M. Substituent Effects Study Reveals the Kinetic Pathway for an Interfacial Reaction. J. Am. Chem. Soc. 2004, 126, 15613-15617.

(3) Williams, J. M.; Rowland, B.; Jeffery, M. T.; Groenewold, G. S.; Appelhans, A. D.; Gresham, G. L.; Olson, J. E. Degradation Kinetics of VX on Concrete by Secondary Ion Mass Spectrometry. Langmuir 2005, 21, 2386-2390.

(4) Li, J.; Thiara, P. S.; Mrksich, M. Rapid Evaluation and Screening of Interfacial Reactions on Self-Assembled Monolayers. Langmuir 2007, 23, 11826-11835.
(5) Orski, S.; Sheppard, G. R.; Arumugam, S.; Arnold, R. M.; Popik, V. V.; Locklin, J. Rate Determination of Azide Click Reactions onto Alkyne Polymer Brush Scaffolds: a Comparison of Conventional and Catalyst-Free Cycloadditions for Tunable Surface Modification. Langmuir 2012, 28, 14693-14702.

(6) Han, C.; Liu, Y.; Ma, J.; He, H. Key Role of Organic Carbon in the Sunlight-Enhanced Atmospheric Aging of Soot by $\mathrm{O}_{2}$. Proc. Natl. Acad. Sci. U. S. A. 2012, 109, 21250-21255.

(7) Näslund, L.-Å. Reaction Kinetics for the Oxygen Hydrogenation Process on $\mathrm{Pt}(111)$ Derived from Temperature-Programmed XPS. Surf. Sci. 2013, 618, 42-48.

(8) Montavon, T. J.; Li, J.; Cabrera-Pardo, J. R; Mrksich, M.; Kozmin, S. A. Three-Component Reaction Discovery Enabled by Mass Spectrometry of Self-Assembled Monolayers. Nat. Chem. 2011, 4, 4551 .

(9) Min, D.-H.; Tang, W.-J.; Mrksich, M. Chemical Screening by Mass Spectrometry to Identify Inhibitors of Anthrax Lethal Factor. Nat. Biotechnol. 2004, 22, 717-720.

(10) Mrksich, M. Mass Spectrometry of Self-Assembled Monolayers: A New Tool for Molecular Surface Science. ACS Nano 2008, 2, 7-18.

(11) Gurard-Levin, Z. A.; Scholle, M. D.; Eisenberg, A. H.; Mrkisch, M. High-Throughput Screening of Small Molecule Libraries using SAMDI Mass Spectrometry. ACS Comb. Sci. 2011, 13, 347-350.

(12) Kuo, H.-Y.; DeLuca, T. A.; Miller, W. M.; Mrksich, M. Profiling Deacetylase Activities in Cell Lysates with Peptide Arrays and SAMDI Mass Spectrometry. Anal. Chem. 2013, 85, 10635-10642.

(13) Diagne, A. B.; Li, S.; Perkowski, G. A.; Mrksich, M.; Thomson, R. J. SAMDI Mass Spectrometry-Enabled High-Throughput Optimization of a Traceless Petasis Reaction. ACS Comb. Sci. 2015, 17, 658-662.

(14) Cody, R. B.; Laramée, J. A.; Durst, H. D. Versatile New Ion Source for the Analysis of Materials in Open Air Under Ambient Conditions. Anal. Chem. 2005, 77, 2297-2302.

(15) Su, J.; Mrksich, M. Using MALDI-TOF Mass Spectrometry to Characterize Interfacial Reactions on Self-Assembled Monolayers. Langmuir 2003, 19, 4867-4870.

(16) Song, L.; Dykstra, A. B.; Yao, H.; Bartmess, J. E. Ionization Mechanism of Negative Ion-Direct Analysis in Real Time: a Comparative Study with Negative Ion-Atmospheric Pressure Photoionization. J. Am. Soc. Mass Spectrom. 2009, 20, 42-50.

(17) Gross, J. H. High-Mass Cluster Ions of Ionic Liquids in PositiveIon and Negative-Ion DART-MS and their Application for WideRange Mass Calibrations. Anal. Bioanal. Chem. 2014, 406, 2853-2862.

(18) Gross, J. H. Analysis of Silicones Released from Household Items and Baby Articles by Direct Analysis in Real Time-Mass Spectrometry. J. Am. Soc. Mass Spectrom. 2015, 26, 511-521.

(19) El-Baba, T. J.; Lutomski, C. A.; Wang, B.; Trimpin, S. Characterizing Synthetic Polymers and Additives using New Ionization Methods for Mass Spectrometry. Rapid Commun. Mass Spectrom. 2014, 28, 1175-1184.

(20) Gross, J. H. Direct Analysis in Real Time-a Critical Review on DART-MS. Anal. Bioanal. Chem. 2014, 406, 63-80.

(21) Kpegba, K.; Spadaro, T.; Cody, R. B.; Nesnas, N.; Olson, J. A. Analysis of Self-Assembled Monolayers on Gold Surfaces using Direct Analysis in Real Time Mass Spectrometry. Anal. Chem. 2007, 79, 5479-5483.

(22) Manova, R. K.; Claassen, F. W.; Nielen, M. W. F.; Zuilhof, H.; van Beek, T. A. Ambient Mass Spectrometry of Covalently Bound Organic Monolayers. Chem. Commun. 2013, 49, 922-924.

(23) Manova, R. K.; Joshi, S.; Debrassi, A.; Bhairamagdi, N. S.; Roeven, E.; Gagnon, J.; Tahir, M. N.; Claassen, F. W.; Scheres, L. M. W.; Wennekes, T.; Schröen, K.; van Beek, T. A.; Zuilhof, H.; Nielen, M. W. F. Ambient Surface Analysis of Organic Monolayers using Direct Analysis in Real Time Orbitrap Mass Spectrometry. Anal. Chem. 2014, 86, 2403-2411.

(24) Zhou, S.; Forbes, M. W.; Abbatt, J. P. D. Application of Direct Analysis in Real Time-Mass Spectrometry (DART-MS) to the Study of Gas-Surface Heterogeneous Reactions: Focus on Ozone and PAHs. Anal. Chem. 2015, 87, 4733-4740. 
(25) Escorihuela, J.Marcelis, A. T. M.Zuilhof, H.Metal-Free Click Chemistry Reactions on Surfaces. Adv. Mater. Interfaces [Online] 10.1002/admi.201500135. 2015.

(26) Manova, R. K.; van Beek, T. A.; Zuilhof, H. Surface Functionalization by Strain-Promoted Alkyne-Azide Click Reactions. Angew. Chem., Int. Ed. 2011, 50, 5428-5430.

(27) Agard, N. J.; Prescher, J. A.; Bertozzi, C. R. Strain-Promoted [3 + 2] Azide-Alkyne Cycloaddition for Covalent Modification of Biomolecules in Living Systems. J. Am. Chem. Soc. 2004, 126, 15046-15047.

(28) Codelli, J. A.; Baskin, J. M.; Agard, N. J.; Bertozzi, C. R. SecondGeneration Difluorinated Cyclooctynes for Copper-Free Click Chemistry. J. Am. Chem. Soc. 2008, 130, 11486-11493.

(29) Ning, X.; Guo, J.; Wolfert, M. A.; Boons, G.-J. Visualizing Metabolically Labeled Glycoconjugates of Living Cells by Copper-Free and Fast Huisgen Cycloadditions. Angew. Chem., Int. Ed. 2008, 47, 2253-2255.

(30) Sletten, E. M.; Bertozzi, C. R. A Hydrophilic Azacyclooctyne for Cu-Free Click Chemistry. Org. Lett. 2008, 10, 3097-3099.

(31) Jewett, J. C.; Sletten, E. M.; Bertozzi, C. R.; Rapid Cu-Free, Click Chemistry with Readily Synthesized Biarylazacyclooctynones. J. Am. Chem. Soc. 2010, 132, 3688-3690.

(32) Friscourt, F.; Ledin, P. A.; Mbua, N. E.; Flanagan-Street, H. R.; Wolfert, M. A.; Street, R.; Boons, G.-J. Polar Dibenzocyclooctynes for Selective Labeling of Extracellular Glycoconjugates of Living Cells. J. Am. Chem. Soc. 2012, 134, 5381-5389.

(33) Beatty, K. E.; Fisk, J. D.; Smart, B. P.; Lu, Y. Y.; Szychowski, J.; Hangauer, M. J.; Baskin, J. M.; Bertozzi, C. R.; Tirrell, D. A. Live-Cell Imaging of Cellular Proteins by a Strain-Promoted Azide-Alkyne Cycloaddition. ChemBioChem 2010, 11, 2092-2095.

(34) Debets, M. F.; van Berkel, S. S.; Schoffelen, S.; Rutjes, F. P. J. T.; van Hest, J. C. M.; van Delft, F. L. Aza-dibenzocyclooctynes for Fast and Efficient Enzyme Pegylation via Copper-Free $(3+2)$ Cycloaddition. Chem. Commun. 2010, 46, 97-99.

(35) Marks, I. S.; Kang, J. S.; Jones, B. T.; Landmark, K. J.; Cleland, A. J.; Taton, T. A. Strain-Promoted "Click" Chemistry for Terminal Labeling of DNA. Bioconjugate Chem. 2011, 22, 1259-1263.

(36) Singh, I.; Freeman, C.; Heaney, F. Efficient Synthesis of DNA Conjugates by Strain-Promoted Azide-Cyclooctyne Cycloaddition in the Solid Phase. Eur. J. Org. Chem. 2011, 2011, 6739-6746.

(37) Wang, C.-F.; Mäkilä, E. M.; Kaasalainen, M. H.; Liu, D.; Sarparanta, M. P.; Airaksinen, A. J.; Salonen, J. J.; Hirvonen, J. T.; Santos, H. A. Copper-free azide-alkyne cycloaddition of targeting peptides to porous silicon nanoparticles for intracellular drug uptake. Biomaterials 2014, 35, 1257-1266.

(38) Laughlin, S. T.; Baskin, J. M.; Amacher, S. L.; Bertozzi, C. R. In Vivo Imaging of Membrane-Associated Glycans in Developing Zebrafish. Science 2008, 320, 664-667.

(39) Canalle, L. A.; van Berkel, S. S.; de Haan, L. T.; van Hest, J. C. M. Copper-Free Clickable Coatings. Adv. Funct. Mater. 2009, 19, $3464-3470$

(40) Orski, S. V.; Poloukhtine, A. A.; Arumugam, S.; Mao, L.; Popik, V. V.; Locklin, J. High Density Orthogonal Surface Immobilization via Photoactivated Copper-Free Click Chemistry. J. Am. Chem. Soc. 2010, 132, 11024-11026.

(41) Manova, R. K.; Pujari, S. P.; Weijers, C. A. G. M.; Zuilhof, H.; van Beek, T. A. Copper-Free Click Biofunctionalization of Silicon Nitride Surfaces via Strain-Promoted Alkyne-Azide Cycloaddition Reactions. Langmuir 2012, 28, 8651-8663.

(42) Wendeln, C.; Singh, I.; Rinnen, S.; Schulz, C.; Arlinghaus, H. F.; Burley, G. A.; Ravoo, B. J. Orthogonal, Metal-Free Surface Modification by Strain-Promoted Azide-Alkyne and Nitrile OxideAlkene/Alkyne Cycloadditions. Chem. Sci. 2012, 3, 2479-2484.

(43) Gobbo, P.; Novoa, S.; Biesinger, M. C.; Workentin, M. S. Interfacial Strain-Promoted Alkyne-Azide Cycloaddition (I-SPAAC) for the Synthesis of Nanomaterial Hybrids. Chem. Commun. 2013, 49, 3982-3984.
(44) Debets, M. F.; van Berkel, S. S.; Dommerholt, J.; Dirks, A. J.; Rutjes, F. P. J. T.; van Delft, F. L. Bioconjugation with Strained Alkenes and Alkynes. Acc. Chem. Res. 2011, 44, 805-815.

(45) Debets, M. F.; Prins, J. S.; Merkx, D.; van Berkel, S. S.; van Delft, F. L. Synthesis of DIBAC Analogues with Excellent SPAAC Rate Constants. Org. Biomol. Chem. 2014, 12, 5031-5037.

(46) Wijdeven, M. A.; Nicosia, C.; Borrmann, A.; Huskens, J.; van Delft, F. L. Biomolecular Patterning of Glass Surfaces via StrainPromoted Cycloaddition of Azides and Cyclooctynes. RSC Adv. 2014, 4, 10549-10552.

(47) Liu, D.; Xu, X.; Su, Y.; He, Z.; Xu, J.; Miao, Q. Self-Assembled Monolayers of Phosphonic Acids with Enhanced Surface Energy for high-Performance Solution-Processed N-Channel Organic Thin-Film Transistors. Angew. Chem., Int. Ed. 2013, 52, 6222-6227.

(48) Pujari, S. P.; Scheres, L.; Marcelis, A. T. M.; Zuilhof, H. Covalent Surface Modification of Oxide Surfaces. Angew. Chem., Int. Ed. 2014, 53, 6322-6356.

(49) Bhairamadgi, N. S.; Pujari, S. P.; Trovela, F. G.; Debrassi, A.; Khamis, A. A.; Alonso, J. M.; Al Zahrani, A. A.; Wennekes, T.; AlTuraif, H. A.; van Rijn, C.; Alhamed, Y. A.; Zuilhof, H. Hydrolytic and Thermal Stability of Organic Monolayers on Various Inorganic Substrates. Langmuir 2014, 30, 5829-5839.

(50) Houseman, B. T.; Mrksich, M. The Microenvironment of Immobilized Arg-Gly-Asp Peptides is an Important Determinant of Cell Adhesion. Biomaterials 2001, 22, 943-955.

(51) Kwon, Y.; Mrksich, M. Dependence of the Rate of an Interfacial Diels-Alder Reaction on the Steric Environment of the Immobilized Dienophile: an example of Enthalpy-Entropy Compensation. J. Am. Chem. Soc. 2002, 124, 806-812.

(52) Collman, J. P.; Devaraj, N. K.; Eberspacher, T. P. A.; Chidsey, C. E. D. Mixed Azide-Terminated Monolayers: a Platform for Modifying Electrode Surfaces. Langmuir 2006, 22, 2457-2464.

(53) Bauer, T.; Schmaltz, T.; Lenz, T.; Halik, M.; Meyer, B.; Clark, T. Phosphonate- and Carboxylate-Based Self-Assembled Monolayers for Organic Devices: a Theoretical Study of Surface Binding on Aluminum Oxide with Experimental Support. ACS Appl. Mater. Interfaces 2013, 5, $6073-6080$

(54) Radhakrishnan, C.; Lo, M. K. F.; Warrier, M. V.; Garcia-Garibay, M. A.; Monbouquette, H. G. Photocatalytic Reduction of an AzideTerminated Self-Assembled Monolayer using CdS Quantum Dots. Langmuir 2006, 22, 5018-5024.

(55) Levine, I.; Weber, S. M.; Feldman, Y.; Bendikov, T.; Cohen, H.; Cahen, D.; Vilan, A. Molecular Length, Monolayer Density, and Charge Transport: Lessons from Al-AlOx/Alkyl-Phosphonate/Hg Junctions. Langmuir 2012, 28, 404-415.

(56) Kosian, M.; Smulders, M. M. J.; Zuilhof, H. Structure and LongTerm Stability of Alkylphosphonic Acid Monolayers on SS316L Stainless Steel. Langmuir 2016, 32, 1047-1057.

(57) Szewczyk, J. W.; Zuckerman, R. L.; Bergman, R. G.; Ellman, J. A. A Mass Spectrometric Labeling Strategy for High-Throughput Reaction Evaluation and Optimization: Exploring C-H Activation. Angew. Chem., Int. Ed. 2001, 40, 216-219.

(58) Cabrera-Pardo, J. R.; Chai, D. I.; Liu, S.; Mrksich, M.; Kozmin, S. A. Label-Assisted Mass Spectrometry for the Acceleration of Reaction Discovery and Optimization. Nat. Chem. 2013, 5, 423-427.

(59) Gouget-Laemmel, A. C.; Yang, J.; Lodhi, M. A.; Siriwardena, A.; Aureau, D.; Boukherroub, R.; Chazalviel, J.-N.; Ozanam, F.; Szunerits, S. Functionalization of Azide-Terminated Silicon Surfaces with Glycans using Click Chemistry: XPS and FTIR Study. J. Phys. Chem. C 2013, 117, 368-375.

(60) Garcia-Hartjes, J.; Dommerholt, J.; Wennekes, T.; van Delft, F. L.; Zuilhof, H. Electronic Effects Versus Distortion Energies during Strain-Promoted Alkyne-Azide Cycloadditions: a Theoretical Tool to Predict Reaction Kinetics. Eur. J. Org. Chem. 2013, 2013, 3712-3720. 